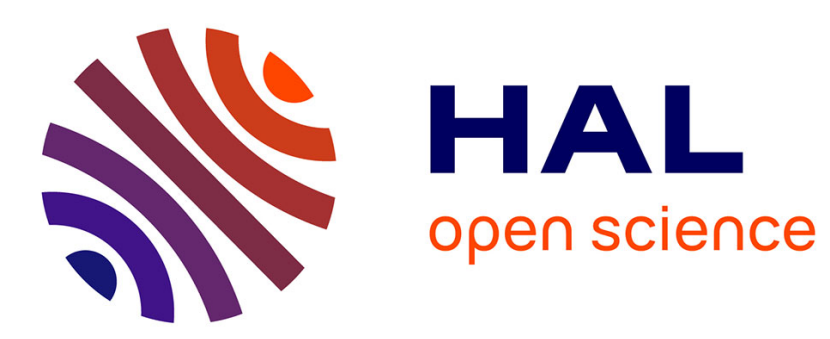

\title{
Spin contrast in SANS of polymers in solution
}

\author{
M.G.D. van Der Grinten, H. Glättli
}

\section{To cite this version:}

M.G.D. van Der Grinten, H. Glättli. Spin contrast in SANS of polymers in solution. Journal de Physique IV Proceedings, 1993, 03 (C8), pp.C8-427-C8-430. 10.1051/jp4:1993887 . jpa-00252318

\section{HAL Id: jpa-00252318 https://hal.science/jpa-00252318}

Submitted on 1 Jan 1993

HAL is a multi-disciplinary open access archive for the deposit and dissemination of scientific research documents, whether they are published or not. The documents may come from teaching and research institutions in France or abroad, or from public or private research centers.
L'archive ouverte pluridisciplinaire HAL, est destinée au dépôt et à la diffusion de documents scientifiques de niveau recherche, publiés ou non, émanant des établissements d'enseignement et de recherche français ou étrangers, des laboratoires publics ou privés. 


\title{
Spin contrast in SANS of polymers in solution
}

\author{
M.G.D. VAN DER GRINTEN and H. GLÄTTLI \\ Commissariat à l'Energie Atomique, Service de Physique de l'Etat Condensé, 91191 Gif-sur-Yvette cedex,
France
}

\begin{abstract}
We present a small angle neutron scattering study of polymers in solution. The contrast between the scattering lengths of the polymer and the solution is created by dynamic polarization of the nuclei in the sample. We used the spectrometer PAON at G5-3 of the LLBSaclay-reactor Orphée which is a prototype of a SANS spectrometer using dynamic nuclear polarization. The power of this method can be seen from the results obtained on two different samples: a solution of the homopolymer polystyrene in orthoterphenyl, and a mixture of two homopolymers dissolved in toluene. We observed for the polystyrene sample the decrease in contrast at positive polarization and increase in contrast for negative polarization. For the sample of the polymer mixture in solution we obtained the different structure factors by taking the SANS spectra for different polarizations. The interference term, obtained separately in the same sample, confirms the mutual interaction.
\end{abstract}

\section{Introduction}

SANS is an often used tool to study macromolecules, like polymers, in solution. For this a difference in scattering length between the polymer and the solution is required. The usual way of obtaining this contrast is using isotopic substitution: one replaces the protons of the solvent by deuterons which have a larger scattering length. An other possibility of creating contrast is using the interaction neutron spin-proton spin. The scattering length of a nucleus is given by

$$
\mathbf{b}=\mathrm{b}_{0}+\mathrm{b}_{n} \mathbf{I} \cdot \mathbf{s}
$$

where $I$ is the nuclear spin and $s$ the neutron spin. It depends on the nuclear polarization as well as on the neutron polarization. With the discovery of the dynamic nuclear polarization in solids, the solid effect [1], it has become possible to obtain high nuclear polarizations up to $100 \%$ [2] [3]. By varying the nuclear polarization one can vary the contrast and suppress or highlight the diffusion of a polymer in solution. This method is very useful in multi-component systems; in a sample consisting of different components one can study these components independently at different polarization. Contrast variation by nuclear polarization has the advantage above isotopic substitution that it offers the possibility to study different components using one single sample. To do this with isotopic substitution one is obliged to make different samples. Results of contrast variation by dynamic nuclear polarization have been reported by several laboratories $[4][5][6]$.

\section{Differential cross-section}

For the case of a homopolymer in solution, the coherent differential cross section can be written as: 


$$
\left(\frac{d \sigma}{d \Omega}\right)_{c o h .}=v^{2} K_{p p}^{2} S_{0}(\mathbf{Q})
$$

$S_{0}(\mathbf{Q})$ is the structure factor of the polymer, for a polymer in a good solvent it is given by the Debye function. The contrast factor is given by:

$$
K_{p p}^{2}=\left(b_{0}^{p}-b_{0}^{s}\right)^{2}+p \bar{P} I\left(b_{n}^{p}-b_{n}^{s}\right)\left(b_{0}^{p}-b_{0}^{s}\right)+\frac{\overline{P^{2}} I^{2}}{4}\left(b_{n}^{p}-b_{n}^{s}\right)^{2}
$$

$\bar{P}$ is the average nuclear polarization and $p$ the neutron polarization. $Q$ is the usual scattering vector $Q=2 \pi / \lambda \sin (2 \theta)$, with $2 \theta$ the scattering angle and $\lambda$ the neutron wavelength. We used the scattering length density $b$ which is defined as the scattering length of a particle per unit volume.

For the case of a mixture of two polymers in solution the differential cross section has three terms:

$$
\left(\frac{d \sigma}{d \Omega}\right)_{c o h .}=v_{1}^{2} K_{p_{1} p_{1}}^{2} S_{0}(\mathbf{Q})+2 v_{1} v_{2} K_{\text {int }}^{2} S_{i n t}(\mathbf{Q})+v_{2}^{2} K_{p_{2} p_{2}}^{2} S_{0}(\mathbf{Q})
$$

The contrast factors $K_{p_{1} p_{1}}^{2}$ and $K_{p_{2} p_{2}}^{2}$ are the contrast factors between the polymer 1 respectively polymer 2 and the solution as defined in (3). $K_{\text {int }}^{2}$ is the interference contrast factor between the two polymers themselves:

$$
\begin{aligned}
K_{i n t}^{2}=\left(b_{0}^{p_{1}}-b_{0}^{s}\right) \cdot\left(b_{0}^{p_{2}}-b_{0}^{s}\right)+ & \frac{p \bar{P} I}{2}\left(\left(b_{n}^{p_{1}}-b_{n}^{s}\right)\left(b_{0}^{p_{2}}-b_{0}^{s}\right)+\left(b_{n}^{p_{2}}-b_{n}^{s}\right)\left(b_{0}^{p_{1}}-b_{0}^{s}\right)\right) \\
& +\frac{\overline{P^{2}} I^{2}}{4}\left(b_{n}^{p_{1}}-b_{n}^{s}\right)\left(b_{n}^{p_{2}}-b_{n}^{s}\right)
\end{aligned}
$$

In figure 1 the different contrast factors of polystyrene (PS) and polydichlorophosphazene (PCIPN) in a partially deuterated solvent are shown as a function of the proton polarization.

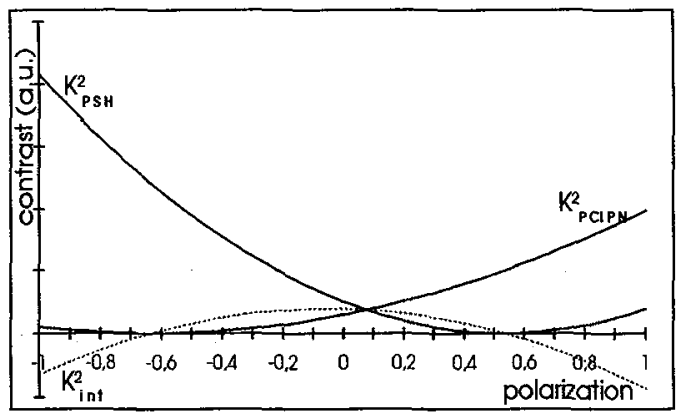

Figure 1: The contrast factors of PS and PCIPN in a solution of $30 / 70$ toluene H/D

\section{Experimental techniques}

In order to obtain a high electron polarization (i.e. $100 \%$ ) and a long nuclear relaxation time, temperatures below $0.5 \mathrm{~K}$ are required. This implies that the sample must be in a dilution refrigerator. $\mathrm{He}^{3}$ is prohibited in the sample environment, due to its enormons absorption cross section for thermal neutrons. We use therefore a dilution refrigerator specially conceived for neutron scattering experiments, where the sample is situated in a cavity separated to the 
dilution chamber. An electromagnet applies a magnetic field of $25 \mathrm{kGauss}$ to the sample, a microwave of $70 \mathrm{GHz}$ induces the dynamic nuclear polarization. The spectrometer PAON has a low intensity $\left(3 \cdot 10^{3} \mathrm{n} \mathrm{s}^{-1}\right.$ at $\lambda=4.7 \AA$, the neutrons polarized by a supermirror). Counting times of 50 hours have been needed for the spectra shown.

The samples are vitreous beads of polymers in solution, obtained by quick freezing of droplets of the solution in liquid nitrogen. Paramagnetic impurities, di-tert-butyl nitroxide, are added to the solution. They provide the free electron spins necessary for the dynamic polarization.

\section{Experimental results}

SANS spectra have been taken from a sample of $2 \%$ PS with a mass of 35.000 in $50 / 50$ OTPH/OTP-D at three polarizations, $P=-50 \%, P=0$ and $P=+50 \%$. Figure 1 shows that the contrast factor of PS is negligible for $P=+50 \%$ and small for $P=0$. At negative polarization the contrast factor has appreciable values. This is still true in 50/50 OTP-H/OPT-D. In figure 2 the SANS spectra are shown for the three polarizations.

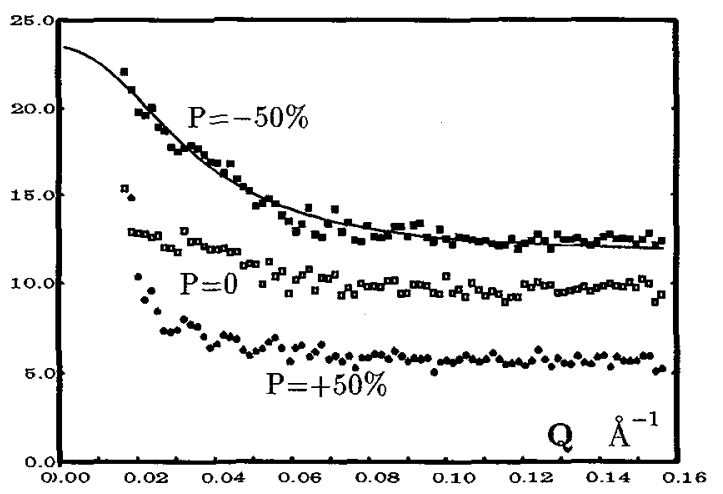

Figure 2: SANS from a sample of $P S$ in 50/50 OTP-H/OTP-D for $P=-50 \%, P=0$ et $P=+50 \%$. The signal of the polymer is strongest for $P=\sim 50 \%$.

As expected from the contrast factors, the scattering is very small for positive and zero polarization. At negative polarization one does clearly see the contribution of PS. This signal has been fitted with a Debye function with a radius of gyration of $R_{g}=47 \AA$, which corresponds to the expected radius of gyration for our polymer at low concentration in a good solvent.

From the sample of the mixture of PS (mass 5.000) and PCIPN (polydispersed) both dissolved at a concentration of $3 \%$ in a solution of $30 / 70$ toluene- $H /$ toluene- $D$, SANS spectra were taken at $P=-38 \%, P=0$ and at $P=+38 \%$. These spectra are shown in figure 3 .

We now see a contribution of the polymers to the scattering at the three different polarizatons. The signal at negative polarization shows the diffusion from PS (here $K_{P C l P N}^{2} \ll K_{P S}^{2}$ ), the signal at positive polarization shows the diffusion from PClPN (here $K_{P S}^{2} \ll K_{P C l P N}^{2}$ ). The scattering at these two polarization gives directly the structure factors of PS respectively PCIPN. At zero polarization the signal is flat, this is due to the negative sign of the interference structure fonction whose contrast factor is comparable to the contrast factors $K_{P C I P N}^{2}$ and $K_{P S}^{2}$ for $P=0$. The negative sign of the interference term corresponds to the model of a mixture of homopolymers in solution taking into account an excluded volume [7].

\section{Conclusion}

We showed the dependence of the scattering intensity of polymers in solution on the nuclear polarization. The signal of PS has been suppressed at positive polarization and enhanced at 


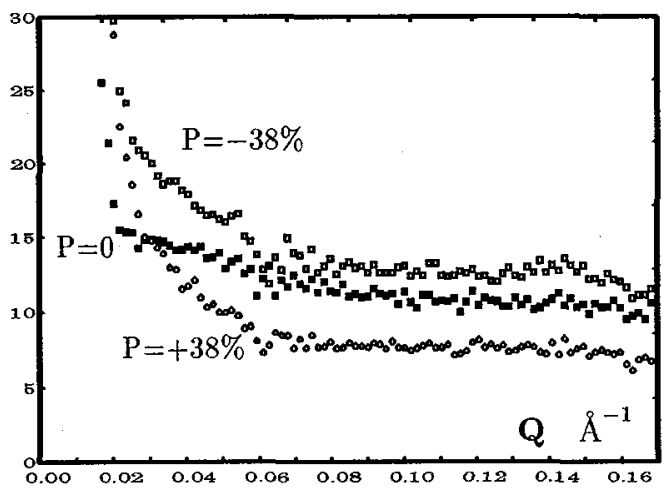

Figure 3: SANS from a sample of PS and PClPN mixed both at 3\% in 30/70 toluene-H/toluene-D for three different polarizations.

negative polarization. The scattering from PS dissolved in OTP follows a Debye function. This indicates that the conformation of the polymer is not altered by our way of making the samples (i.e. quick freezing).

The scattering from the mixture of two homopolymers in solution showed the (negative) interference term in the same $Q$ region as the structure functions of the homopolymers themselves. This means that the mean distance between the different homopolymers is of the same order as their radius of gyration.

\section{References}

[1] A. Abragam and W.G. Proctor, C.R. Acad. Sci. Paris 246, 2253 (1958)

[2] W. de Boer and T.O. Niinikoski, Nucl. Instr. Meth. 114495 (1974)

[3] T.O. Niinikoski and J.M. Rieubland, Phys. Lett. 72a 141 (1979)

[4] W. Knop, M. Hirai, G. Olah, W. Meerwinck, H.J. Schink, H.B. Stuhrmann, R. Wagner, M. WenkowEsSouni, J. Zhao, O. Schärpf, R.R. Crichton, M. Krumpolc, K.H. Nierhaus, T.O. Niinikoski and A. Rijllart, Physica B ,173 (1991)

[5] M. Kohgi, M. Ishida, Y. Ishikawa, S. Ishimoto, Y. Kanno, A. Masaike, Y. Masuda and K. Morimoto, J. Phys. Soc. Japan, 562682 (1987)

[6] C. Fermon, H. Glättli, M. van der Grinten, M. Eisenkremer and M. Pinot, Physica B 180 \& 181, 991 (1992)

[7] H. Benoît and M. Benmouna, Macromolecules 17, 535 (1984) 